\title{
Correction to: Optimal Planning of Asian Expressway Network with Dynamic Interregional Input-Output Programming Model
}

\section{Correction to:}

\author{
Chapter 7 in: H. Kohno, Y. Higano,
}

Public Investment Criteria, New Frontiers in Regional

Science: Asian Perspectives 2,

https://doi.org/10.1007/978-4-431-55221-5_7

This chapter was inadvertently published with incorrect preposition in the title which has now been corrected to "Optimal Planning of Asian Expressway Network with Dynamic Interregional Input-Output Programming Model”. 